\title{
Radio continuum surveys: lessons learned
}

\author{
J. J. Condon* \\ National Radio Astronomy Observatory, ${ }^{\dagger} 520$ Edgemont Road, Charlottesville, VA 22903 USA \\ E-mail: jcondon@nrao.edu
}

While making and using radio continuum surveys, radio astronomers have had many learning experiences, as in "Oh, no! Not another learning experience!" They include problems with confusion from multiple sources in each beam area, insensitivity to low-brightness sources, correcting source counts for partially resolved or missing sources, and ignoring systematic errors, particularly the consequences of limited dynamic range.

The principal lessons that I have learned from these experiences are:

(1) The universe is not a vacuum. The design of surveys (and survey telescopes) should take into account known source properties, not just purely instrumental parameters such as "survey speed." (2) The radiometer equation and Gaussian statistics are insensitive to the most insidious problems that plague radio continuum surveys. More sophisticated mathematical analyses are needed to optimize telescope and survey design.

(3) Never forget the difference between flux density ( Jy) and peak flux density (Jy beam ${ }^{-1}$ or $\mathrm{K}$ ), which is a surface brightness. Ideally a survey is complete to some flux density (a source property), but all survey images are sensitivity limited by surface brightness (Jy beam ${ }^{-1}$ ), which depends on the instrumental beam solid angle.

(4) Resolution corrections to counts of faint sources are so large and poorly understood that they limit the usefulness of sub-mJy surveys.

These lessons are applied to design a possible Jansky Very Large Array (VLA) "wedding cake" survey that could provide a strong extinction-free constraint on the cosmological evolution of the star formation rate over the redshift range $0 \leq z \lesssim 3$ in which most stars were formed.

EXTRA-RADSUR2015 (*)

20-23 October 2015

Bologna, Italy

(*) This conference has been organized with the support of the Ministry of Foreign Affairs and International Cooperation, Directorate General for the Country Promotion (Bilateral Grant Agreement ZA14GR02 - Mapping the Universe on the Pathway to SKA)

\footnotetext{
* Speaker.

${ }^{\dagger}$ The National Radio Astronomy Observatory is a facility of the National Science Foundation operated under cooperative agreement by Associated Universities, Inc.
} 


\section{Introduction}

This review covers the main lessons I have learned from 50 years of reading survey papers, making surveys, refereeing survey proposals and manuscripts, and talking with other astronomers. Section 2 is an overview of the radio sky. The source density sets the "confusion" (Section 3) level and the required survey dynamic range (Section 4). Survey angular resolution $\theta$ is a compromise between avoiding confusion and maintaining the brightness sensitivity needed to detect dim sources, especially star-forming galaxies (Section 5). Currently sub-mJy surveys and their source counts are limited by poorly understood corrections for partial resolution (Section 6). Section 7 presents a "wedding cake" survey designed to study the cosmological evolution of star formation.

\section{The radio sky}

Almost all radio continuum sources are extragalactic and very distant. The top half of Figure 1 is an equal-area polar sky projection showing the positions of all NVSS [7] sources with $S>$ $100 \mathrm{mJy}$ in the $82 \%$ of the sky north of $\delta=-40^{\circ}$. Our Galaxy is visible only as a narrow band near the Galactic center at $\alpha \approx 18^{\mathrm{h}}, \delta \approx-30^{\circ}$. The bottom half shows the locations of all sources with $S>2.5 \mathrm{mJy}$ and $\delta>+75^{\circ}$. The extremely isotropic sky distributions of both strong and weak extragalactic sources implies that their average nearest-neighbor separation is much bigger than the 5-10 Mpc galaxy correlation length, so most of them must be at cosmological redshifts.

The $1.4 \mathrm{GHz}$ flux-density distribution of radio sources is plotted in Figure 2, after division by the $n(S) \propto S^{-5 / 2}$ source count of a nonevolving static Euclidean universe. This historic normalization is appropriate only for sources at low redshifts $z \ll 1$. Radio sources occupy the evolving, expanding, and non-Euclidean part of the universe, so the actual source count is not a horizontal line. Evolutionary models $[4,13]$ separate the total source count (continuous curve) into counts of sources powered primarily by AGNs (dashed curve) or by star formation (dotted curve).

In any cosmology, the sky brightness $d T_{\mathrm{b}}$ contributed by sources in the flux-density range $S$ to $S+d \log (S)$ at frequency $v$ is

$$
\left[\frac{d T_{\mathrm{b}}}{d \log (S)}\right]=\left[\frac{\ln (10) c^{2}}{2 k_{\mathrm{B}} v^{2}}\right] S^{2} n(S) .
$$

Figure 3 [9] shows the same evolutionary model curves plotted as $S^{2} n(S)$ instead of $S^{5 / 2} n(S)$, so the right ordinate now indicates the contributions $d T_{\mathrm{b}}$ to sky brightness by the AGN and starforming source populations. In this figure, the data points and "confusion" count box are actually $\lambda=160 \mu \mathrm{m}$ results [2] converted to $1.4 \mathrm{GHz}$ by the median FIR/radio flux-density ratio $\langle q\rangle=$ $\log \left(S_{160 \mu \mathrm{m}}\right) / \log \left(S_{1.4 \mathrm{GHz}}\right)=2.5$ of star-forming galaxies, confirming the model prediction that star-forming galaxies dominate the radio sky below $S_{1.4 \mathrm{GHz}} \sim 0.3 \mathrm{mJy}$.

The models of radio source evolution are really quite simple [5]. If $\rho(L)$ is the comoving space density of radio sources with spectral luminosities between $L$ and $L+d L$, their spectral energy per unit volume is $u(L) \equiv L \rho(L)$. The logarithmic luminosity function defined by $\rho_{\mathrm{m}} \equiv \ln (m) L \rho(L)$ is the space density of sources in the logarithmic luminosity interval $m$; e.g., $m \equiv \operatorname{dex}(0.4)=1$ "magnitude," and the corresponding logarithmic spectral energy per unit volume is $u_{\mathrm{m}}(L) \equiv L \rho_{\mathrm{m}}(L)$. Figure 4 shows $u_{\mathrm{m}}(L)$ at $1.4 \mathrm{GHz}$ for low-redshift radio sources powered 


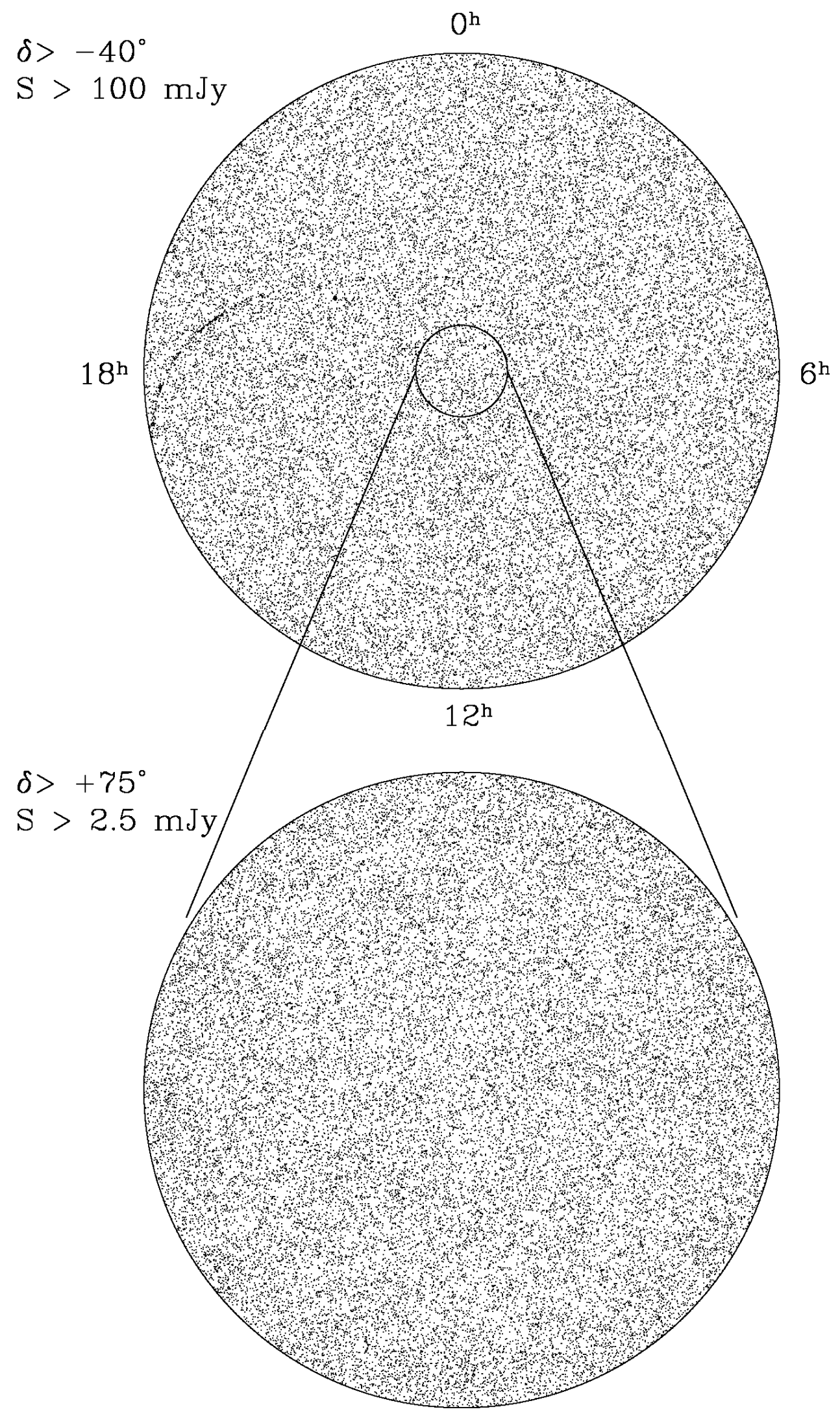

Figure 1: Almost all radio sources are extragalactic, isotropically distributed on the sky, and very distant. 


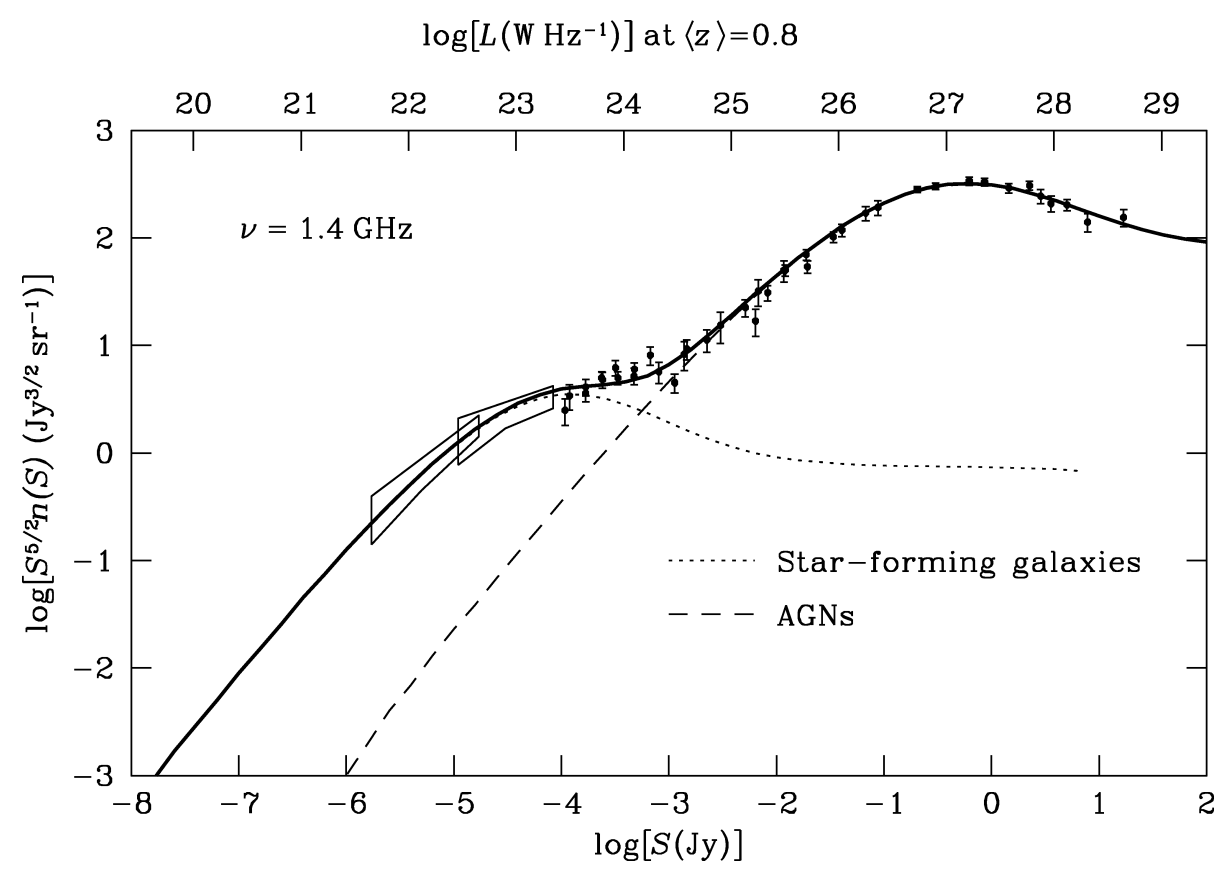

Figure 2: The static-Euclidean-normalized differential source counts $S^{5 / 2} n(S)$ (data points from individual source detections, boxes representing "confusion" by sources too weak to be resolved and detected individually, and curves from the evolutionary models) are shown as a function of $1.4 \mathrm{GHz}$ flux density $S$ on the lower abscissa. The corresponding spectral luminosities $L$ of sources at their median redshift $\langle z\rangle \sim 0.8$ are indicated on the upper abscissa.

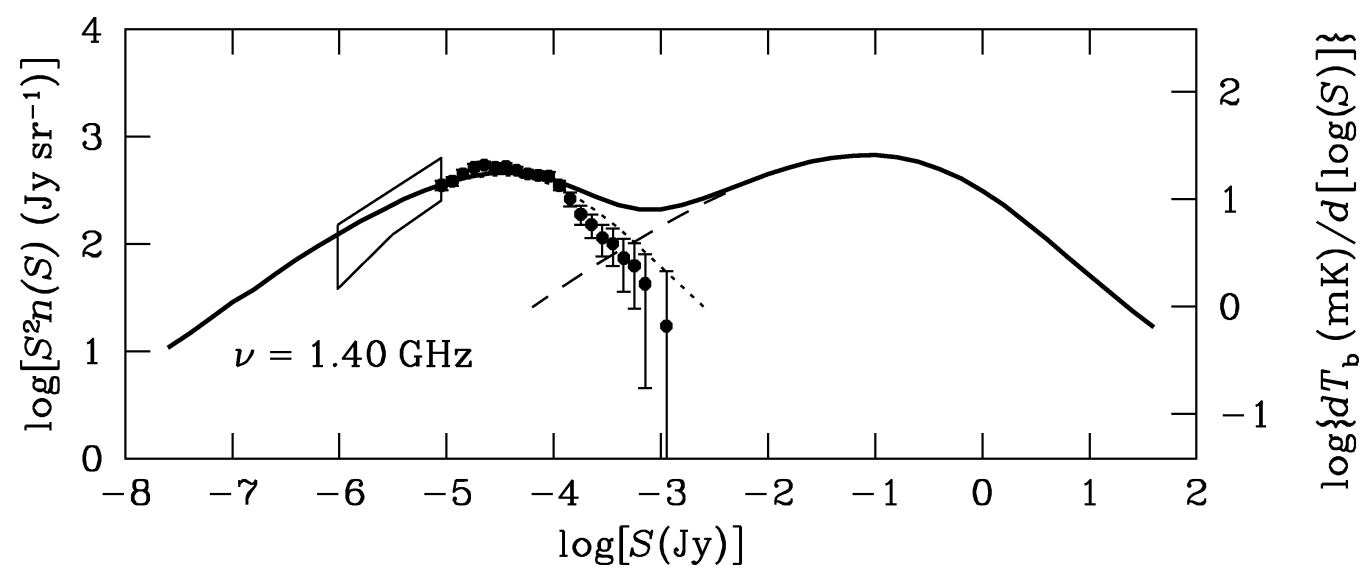

Figure 3: The model source counts $S^{5 / 2} n(S)$ shown in Figure 2 are replotted here with the normalization $S^{2} n(S)$ (left ordinate) that is proportional to the sky brightness contribution $d T_{\mathrm{b}}$ per decade of flux density (right ordinate). The data points and "confusion" count box show the $\lambda=160 \mu \mathrm{m}$ counts [2] converted to $1.4 \mathrm{GHz}$ by the median FIR/radio ratio of star-forming galaxies. 
by star-forming galaxies and by AGNs [8]. Although the space densities $\rho_{\mathrm{m}}(L)$ of both populations increase monotonically to the lowest observed luminosities, their energy densities $u_{\mathrm{m}}(L)$ have smooth but well-defined peaks near $\log \left[L_{1.4 \mathrm{GHz}}\left(\mathrm{W} \mathrm{Hz}^{-1}\right)\right]=22$ for star-forming galaxies and $\log \left[L_{1.4 \mathrm{GHz}}\left(\mathrm{W} \mathrm{Hz}^{-1}\right)\right]=25$ for AGNs. By analogy with Olbers' paradox, $u_{\mathrm{m}}(L)$ for any source population with a finite energy density must peak at some finite luminosity. Sources with luminosities near the peak contribute the most to the sky brightness produced by that population. In any narrow redshift range, the brightness-weighted source count $S^{2} n(S)$ is proportional to $u_{\mathrm{m}}(L)$. The combination of strong $(10 \times)$ luminosity evolution and the large $K$-correction for steep-spectrum radio sources $(\langle\alpha\rangle \approx-0.7)$ effectively confines most sources to a fairly thin "shell" with the same median redshift $\langle z\rangle \sim 0.8$ over a wide flux-density range [5]. Consequently, the curves for starforming galaxies and AGNs in Figure 3 are slightly smoothed versions of the curves in Figure 4.

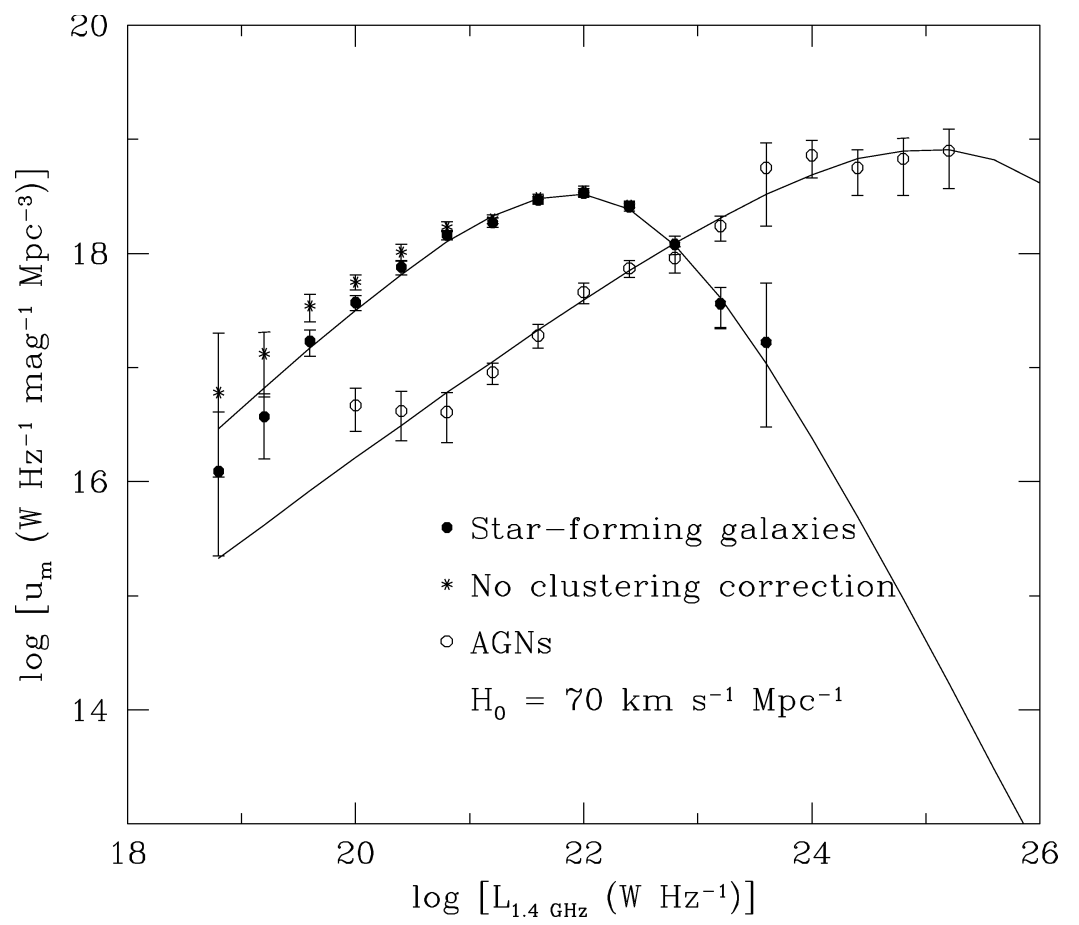

Figure 4: The logarithmic spectral energy densities $u_{\mathrm{m}}(L)$ of radio sources powered by star formation (filled circes) and by AGNs (open circles) look like the brightness-weighted counts $S^{2} n(S)$ in Figure 3.

\section{Confusion}

As many radio astronomers have learned the hard way, multiple faint sources stronger than the rms image noise $\sigma_{\mathrm{n}}$ may overlap in a single telescope beam and cause "confusion" fluctuations with rms $\sigma_{\mathrm{S}}>\sigma_{\mathrm{n}}$ in image peak flux density (meaning, flux density per beam solid angle) or rms $\sigma_{\mathrm{T}}>\sigma_{\mathrm{n}}$ in brightness temperature. The confusion amplitude distribution is not Gaussian; it has a long positive tail [3], so sources fainter than 5 times the rms confusion should not be trusted. Figure 5 plots the rms confusion at $1.4 \mathrm{GHz}$ versus the FWHM $\theta$ of a Gaussian beam. For example, the upcoming EMU survey [12] with $\theta=10^{\prime \prime}$ should have $\sigma_{\mathrm{S}} \approx 31 \times 10^{2} \mathrm{nJy} \mathrm{beam}^{-1}=$ $3.1 \mu \mathrm{Jy}$ beam $^{-1}$. Values in Figure 5 can be scaled to other frequencies by $\sigma_{\mathrm{S}} \propto v^{-0.7}$ or $\sigma_{\mathrm{T}} \propto v^{-2.7}$. 
Thus the $3 \mathrm{GHz}$ rms confusion in the $\theta=8^{\prime \prime}$ beam of the VLA C configuration is $\sigma_{\mathrm{S}} \approx 27 \times 8^{2} \times$ $(3 / 1.4)^{-0.7} \mathrm{nJy} \mathrm{beam}^{-1} \approx 1.0 \mu \mathrm{Jy}_{\text {beam }}{ }^{-1}[9]$.

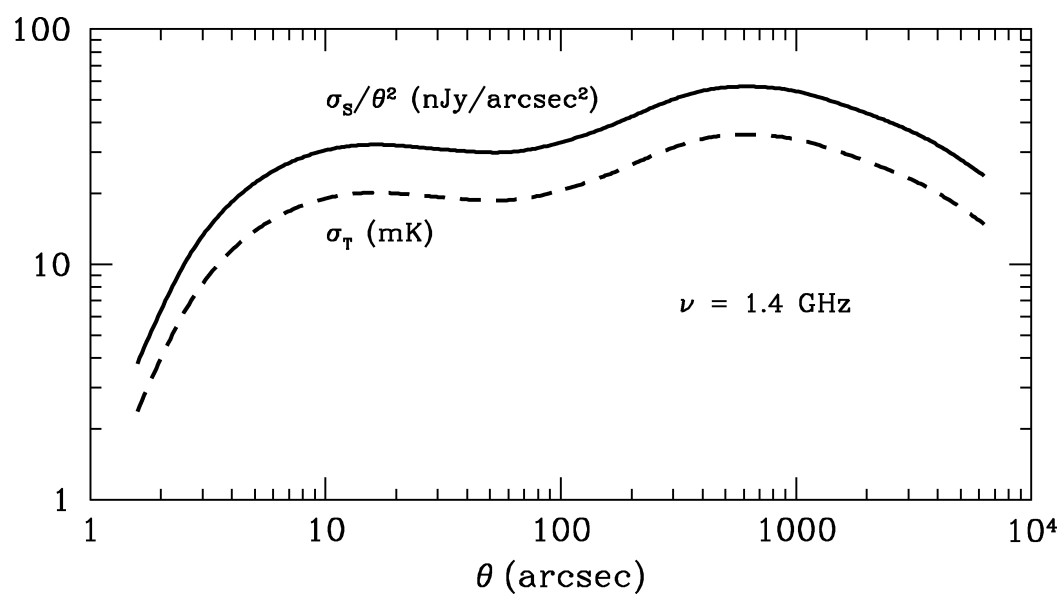

Figure 5: The rms confusion at $1.4 \mathrm{GHz}$ is shown as a peak flux density $\left(\mathrm{nJy} \mathrm{arcsec}^{-2}\right.$ ) and as brightness temperature (mK). It scales as $\sigma_{\mathrm{S}} \propto v^{-0.7}$ and $\sigma_{\mathrm{T}} \propto v^{-2.7}$ and falls sharply for beams smaller than $\theta \approx 10^{\prime \prime}$.

Fortunately, the dropoff of $S^{2} n(S)$ below $S \approx 10 \mu \mathrm{Jy}$ at $1.4 \mathrm{GHz}$ means that confusion should not limit the sensitivity of future arrays such as the SKA [6]. At microJy flux densities, the confusion distribution is so skewed that it is not well described by its rms, and better way to describe the confusion limit is by the number of beam solid angles $\Omega_{\mathrm{b}}=\pi \theta^{2} /(4 \ln 2)$ per source at that peak flux density; e.g., $25 \Omega_{\mathrm{b}}$ source $^{-1}$. The upper curve in Figure 6 indicates that $\theta \lesssim 1^{\prime \prime}$ will not be confusion limited for point sources as faint as $S_{1.4 \mathrm{GHz}} \sim 0.01 \mu \mathrm{Jy}$.

\section{Survey Dynamic Range}

The dynamic range $D$ of an image was originally defined as the ratio of the highest brightness to the rms noise, $D=S_{\max } / \sigma_{\mathrm{n}}$, which is appropriate for an image dominated by a strong point source at its center. For a sky survey image containing many sources scattered throughout the primary beam, $S_{\max }$ should be replaced by the quadratic sum over all sources $\left(\sum_{i} S_{i}^{2}\right)^{1 / 2}$ in the primary beam. Also, small telescope tracking errors cause a larger degradation of survey dynamic range. To exploit its low rms noise $\sigma_{\mathrm{n}} \approx 9 \mathrm{nJy}_{\text {beam }}{ }^{-1}$ after $\tau=10^{6} \mathrm{~s}$ of integration and even lower confusion at $1.4 \mathrm{GHz}$, the SKA will need an unprecedented survey dynamic range $D \sim$ $2 \times 10^{7} \sim 73 \mathrm{db}$ [6]. Existing $1.4 \mathrm{GHz}$ surveys reach only $D \lesssim 40 \mathrm{db}$. The NVSS is actually dynamic range limited, for example, with $\sigma \sim 0.45 \mathrm{mJy} \mathrm{beam}^{-1}$ in total intensity being larger than $\sigma \sim 0.29 \mathrm{mJy}^{-1}$ beam $^{-1}$ in Stokes $Q, U$, and $V$. Especially at long wavelengths, where sources are stronger and primary beams are larger, the telescope performance requirements (pointing accuracy, primary beamshape stability on the sky, sidelobe level, ...) for sensitive sky surveys are very strict.

\section{Brightness Sensitivity}

The dimensions of intensity in any astronomical image are flux density per solid angle (e.g., units of Jy beam ${ }^{-1}$, MJy sr ${ }^{-1}$, or K), not flux density (Jy). Thus it is correct to say that that the 
NVSS detection limit is $2.4 \mathrm{mJy}_{\text {beam }}^{-1}$ or $0.7 \mathrm{~K}$, but saying that it is $S \approx 2.4 \mathrm{mJy}$ is misleading and is true only to the extent that the NVSS beam $\left(\theta=45^{\prime \prime}\right)$ is bigger than most 2.4 mJy sources. Sources powered by star formation in galaxies have a median brightness temperature [11]

$$
\frac{\left\langle T_{\mathrm{b}}\right\rangle}{\mathrm{K}} \approx 2.5\left(\frac{v}{\mathrm{GHz}}\right)^{-2.7},
$$

or $\left\langle T_{\mathrm{b}}\right\rangle \sim 1 K$ at $v=1.4 \mathrm{GHz}$. Thus the NVSS $\left(5 \sigma_{\mathrm{n}} \approx 0.7 \mathrm{~K}\right)$ and EMU $\left(5 \sigma_{\mathrm{n}} \approx 0.3 \mathrm{~K}\right)$ have the brightness sensitivity needed to detect normal star-forming galaxies, but FIRST [1] $\left(5 \sigma_{\mathrm{n}} \approx 16 \mathrm{~K}\right)$ does not, no matter how high their flux densities $S$ may be.

\section{Corrections for Partial Resolution and Missing Sources}

Although dozens of fields have been imaged with detection limits $\lesssim 0.1 \mathrm{Jy}$ beam $^{-1}$, they yield source counts below $S \sim 1$ mJy that disagree by several times the combined Poisson counting, clustering, and noise errors [10]. The scatter among fields observed with one instrument and analyzed by one author is much smaller, so it appears that the fault lies not in our stars, but in ourselves. Different authors observing with different angular resolutions make different count corrections to compensate for partially resolved and missing sources whose flux densities in mJy are higher than their peak flux densities in $\mathrm{mJy}$ beam $^{-1}$, and the errors induced by these corrections appear to be much larger than the error estimates. Until this problem is understood and the correct error expressions are derived, it will remain the biggest limitation faint-source surveys.

\section{A "Wedding Cake" Survey of Star-forming Galaxies}

For any observing time $\tau$, the radiometer equation forces a tradeoff between rms noise $\sigma_{\mathrm{n}}$ and survey sky coverage: $\Omega \propto \sigma_{\mathrm{n}}^{2} \tau$. The cumulative sky density $N(>S)$ of radio sources never varies as rapidly as $S^{-2}$, so the number of sources detected by a "service" survey like the NVSS [7] is maximized by covering the largest $\Omega$ with uniform but high $\sigma_{\mathrm{n}}$. However, many astrophysical problems require statistically useful samples of sources too faint to be detected in all-sky surveys. A "wedding cake" survey of several layers, each with a different $\sigma_{\mathrm{n}}$ and $\Omega$, is better for problems such as tracing the evolving SFRD (star formation rate density, or mass of stars formed per unit time per unit comoving volume). Optimizing the wedding cake profile actually depends more on calculus than on astrophysics, so this example can easily be modified for other science goals.

To minimize Poisson counting errors, the wedding-cake survey should yield large numbers $N \gg N^{1 / 2}$ of sources (e.g., $N \sim 10^{2}$ gives fractional errors $N^{1 / 2} / N \sim 0.1$ ) in each relevant luminosity and redshift bin. The radio spectral luminosity $L$ of a star-forming galaxy is proportional to its star-formation rate, so the "relevant" bins should sample all luminosity and redshift ranges that produce most of the radio sky brightness $T_{\mathrm{b}}$ contributed by star-forming galaxies. Sources stronger than $S_{\min } \approx 1 \mu \mathrm{Jy}$ at $v \approx 3 \mathrm{GHz}$ account for about $89 \%$ of the star-forming galaxy contribution [9], so a good SFRD wedding cake should reach this depth. The VLA is badly limited by dynamic range at $L$ band $(1<v \mathrm{GHz}<2)$ but not at $S$ band $(2<v \mathrm{GHz}<4)$, so $S$ band is better for the wedding cake. The effective $S$ band center frequency is $\langle v\rangle \approx 2.7 \mathrm{GHz}$. Star-forming galaxies have 
Table 1: Star-forming galaxy counts at $2.7 \mathrm{GHz}$

\begin{tabular}{ccccc}
\hline \hline Layer & $\begin{array}{c}\log \left(S_{\min }\right) \\
(\mathrm{Jy})\end{array}$ & $\begin{array}{c}\log \left(S_{\max }\right) \\
(\mathrm{Jy})\end{array}$ & $\begin{array}{c}S^{2} n(S) \\
\left(\mathrm{Jy} \mathrm{sr}^{-1}\right)\end{array}$ & $\begin{array}{c}\Delta N \\
\left(\mathrm{sr}^{-1}\right)\end{array}$ \\
\hline 1 & -6.0 & -5.6 & 129 & $7.52 \times 10^{7}$ \\
2 & -5.6 & -5.2 & 194 & $4.55 \times 10^{7}$ \\
3 & -5.2 & -4.8 & 256 & $2.41 \times 10^{7}$ \\
4 & -4.8 & -4.4 & 275 & $1.05 \times 10^{7}$ \\
5 & -4.4 & -4.0 & 221 & $3.50 \times 10^{6}$ \\
6 & -4.0 & -3.6 & 129 & $8.60 \times 10^{5}$ \\
7 & -3.6 & -3.2 & 59 & $1.64 \times 10^{5}$ \\
\hline
\end{tabular}

a smooth $u_{\mathrm{m}}(L)$ distribution (Figure 4 ), so about seven luminosity bins of width $\operatorname{dex}(0.4)$ are sufficient to sample and needed to span the luminosity range $\Delta \log (L) \sim 3$ of the star-forming galaxies that account for most of the local SFRD.

In any narrow redshift range, the flux density of a source is proportional to its luminosity, so the brightness-weighted differential source count $S^{2} n(S) \propto L^{2} \rho(L)$ contributed by any source population has a peak at some flux density that depends on redshift and the amount of luminosity evolution. Roughly speaking, radio sources associated with both star-forming galaxies and AGNs have median redshifts $\langle z\rangle \sim 0.8$ and evolved luminosities $\sim 10 \times$ their local luminosities [5], so the brightness-weighted $1.4 \mathrm{GHz}$ source count (Figure 3) has two peaks, at $\log [S(\mathrm{Jy})] \approx-4.4$ (star-forming galaxies) and -1 (AGNs). Because the effective spectral index of these sources is $\langle\alpha\rangle \approx-0.7$, the peaks at $v \approx 2.7 \mathrm{GHz}$ are near $\log [S(\mathrm{Jy})] \approx-4.6$ and -1.2 , respectively.

To follow SFRD evolution well, a $v \approx 2.7 \mathrm{GHz}$ wedding-cake survey should at least span the logarithmic flux-density range $-6<\log [S(\mathrm{Jy})]<-3$, divided into $\eta \sim 3 / 0.4 \sim 7$ logarithmic flux-density layers of thickness $m=\operatorname{dex}(0.4)$ as listed in Table 1. Columns 2 and 3 list the fluxdensity range best covered by each layer, $S^{2} n(S)$ is evaluated at the middle of the layer [e.g., $\log (S)=-5.8$ for Layer 1], and $\Delta N$ is the expected number per steradian of star-forming galaxies with $S_{\min }<S<S_{\max }$ [4].

Because the derivative of a smooth function is zero near its peak, the differential source count is roughly $n(S) \propto S^{-2}$ near the $S^{2} n(S)$ peak, and the number of sources per unit solid angle in each logarithmic flux-density range is proportional to $S^{-1}$. To yield about the same number $\Delta N$ of sources in each layer of an ideal wedding-cake survey,

(1) the layer solid angle $\Omega$ should be inversely proportional to its sensitivity limit $S_{\min }$, and

(2) each layer should be fairly thin (small $\mathrm{m}$ ) to ensure that sources are distributed uniformly throughout the layer.

These two rules follow from calculus alone, so they should apply to most wedding-cake surveys.

If $\Omega \propto S_{\min }$, then each layer needs $\Delta \tau \propto \Omega S_{\min } \propto S_{\min }^{-1}$ observing time. The total time $\tau$ for a survey with $\eta$ layers of fixed logarithmic thickness $S_{\max } / S_{\min }=m$ is

$$
\tau=\sum_{\mathrm{i}=1}^{\eta}(\Delta \tau)_{\mathrm{i}}=(\Delta \tau)_{1} \sum_{\mathrm{i}=0}^{\eta-1}\left(\frac{1}{m}\right)^{i}=(\Delta \tau)_{1}\left[\frac{1-m^{-\eta}}{1-m^{-1}}\right] .
$$


Table 2: A VLA $2.7 \mathrm{GHz}$ wedding-cake survey for star-forming galaxies

\begin{tabular}{|c|c|c|c|c|c|c|c|}
\hline Layer & $\begin{array}{c}\sigma_{\mathrm{n}}=S_{\min } / 5 \\
\left(\mu \mathrm{Jy}_{\text {beam }}^{-1}\right)\end{array}$ & $\begin{array}{c}\theta \\
(\operatorname{arcsec})\end{array}$ & $\begin{array}{c}\sigma_{\mathrm{c}} \\
\left(\mu \mathrm{Jy}_{\text {beam }}^{-1}\right)\end{array}$ & $N_{\mathrm{p}}$ & $\begin{array}{c}\Delta \tau \\
\text { (hours) }\end{array}$ & $\begin{array}{c}\Omega \\
\left(\mathrm{deg}^{2}\right)\end{array}$ & $\Delta N$ \\
\hline 1 & 0.20 & 2.5 & 0.04 & 1 & 860 & 0.044 & $1.0 \times 10^{3}$ \\
\hline 2 & 0.50 & 2.5 & 0.04 & 1 & 138 & 0.044 & $6.1 \times 10^{2}$ \\
\hline 3 & 1.26 & 2.5 & 0.04 & 3 & 66 & 0.132 & $9.7 \times 10^{2}$ \\
\hline 4 & 3.17 & 8 & 1.1 & 7 & 24 & 0.31 & $9.8 \times 10^{2}$ \\
\hline 5 & 7.96 & 8 & 1.1 & 19 & 10.3 & 0.83 & $8.9 \times 10^{2}$ \\
\hline 6 & 20.0 & 24 & 12 & 91 & 7.8 & 4.0 & $1.0 \times 10^{3}$ \\
\hline 7 & 50.0 & 24 & 12 & 500 & 6.9 & 22.0 & $1.1 \times 10^{3}$ \\
\hline
\end{tabular}

For $m=\operatorname{dex}(0.4)$ and $\eta=7, \tau \approx 1.66(\Delta \tau)_{1}$; that is, the most sensitive layer needs more time $(\Delta \tau)_{1}$ than all of the other layers combined. This conclusion actually holds for any $m \geq 2$ and $\eta<\infty$.

Table 2 suggests a practical S-band VLA survey matched to the star-forming galaxy population in Table 1. For each layer, an SNR $=5$ point-source detection limit implies that the rms noise $\sigma_{\mathrm{n}}=S_{\min } / 5$ (Column 2). Star-forming galaxies aren't point sources, so $\theta$ must be large enough to satisfy their brightness-temperature detection limit (see Equation 5.1):

$$
\left(\frac{\theta}{\operatorname{arcsec}}\right)^{2} \geq 2.44\left(\frac{\sigma_{\mathrm{n}}}{\mu \mathrm{Jy} \mathrm{beam}^{-1}}\right)\left(\frac{v}{\mathrm{GHz}}\right)^{+0.7} .
$$

With "robust" weighting, the VLA B-, C-, and D-configurations provide $\theta \approx 2.5^{\prime \prime}, 8$ ", and 24 ", respectively, at $2.7 \mathrm{GHz}$. Column 3 of Table 2 lists for each layer the angular resolution of the largest array that provides sufficient surface-brightness sensitivity to detect most star-forming galaxies. The rms confusion levels derived from Figure 5 are listed in Column 4; they are all much smaller than the rms noise. Figure 6 shows as a function of $S_{\min }$ the narrow range of beamwidths $\theta$ in which star-forming galaxies can be detected and the $\left.\left(S_{\min }, \theta\right)\right)$ points of the seven layers. The take-away lesson from Columns 1-4 of Table 2 is that the VLA is uniquely capable of making a weddingcake survey of star-forming galaxies because it is re-configurable. No single VLA configuration is suitable for all layers, nor is any comparable fixed array such as ASKAP, WSRT, GMRT, ...

The effective field-of-view covered by each VLA pointing at $2.7 \mathrm{GHz}$ is $\Omega_{\mathrm{FoV}} \approx 0.044 \mathrm{deg}^{2}$. Column 7 of Table 2 lists the total solid angle $\Omega=N_{\mathrm{p}} \Omega_{\mathrm{FoV}}$ covered by the $N_{\mathrm{p}}$ pointings going into each layer, and Column 8 lists the expected number $\Delta N$ of star-forming galaxies in each layer. Figure 7 shows the survey coverage of the luminosity-redshift plane. The seven survey layers cover the seven flux-density bands bounded by the eight diagonal dotted lines at $2.7 \mathrm{GHz}$ flux densities $\log [S(\mathrm{Jy})]=-3.2$ (top) to -6.0 (bottom) listed in Table 1 . There are $\Delta N \sim 10^{3}$ star-forming galaxies in each band. The upward arrows indicate that the largest layers also detect significant numbers of galaxies stronger than $\log [S(\mathrm{Jy})]=-3.2$. The thick curve is the evolving luminosity corresponding to the peak of $u_{\mathrm{m}}(L, z)$ derived from the $1.4 \mathrm{GHz}$ local radio luminosity function [8] converted to $2.7 \mathrm{GHz}$ via $\langle\alpha\rangle=-0.7$ and evolved as specified in Table 1 of [4]; that is, the thick curve is near the middle of the luminosity range reflecting the highest SFRD at each redshift. The 


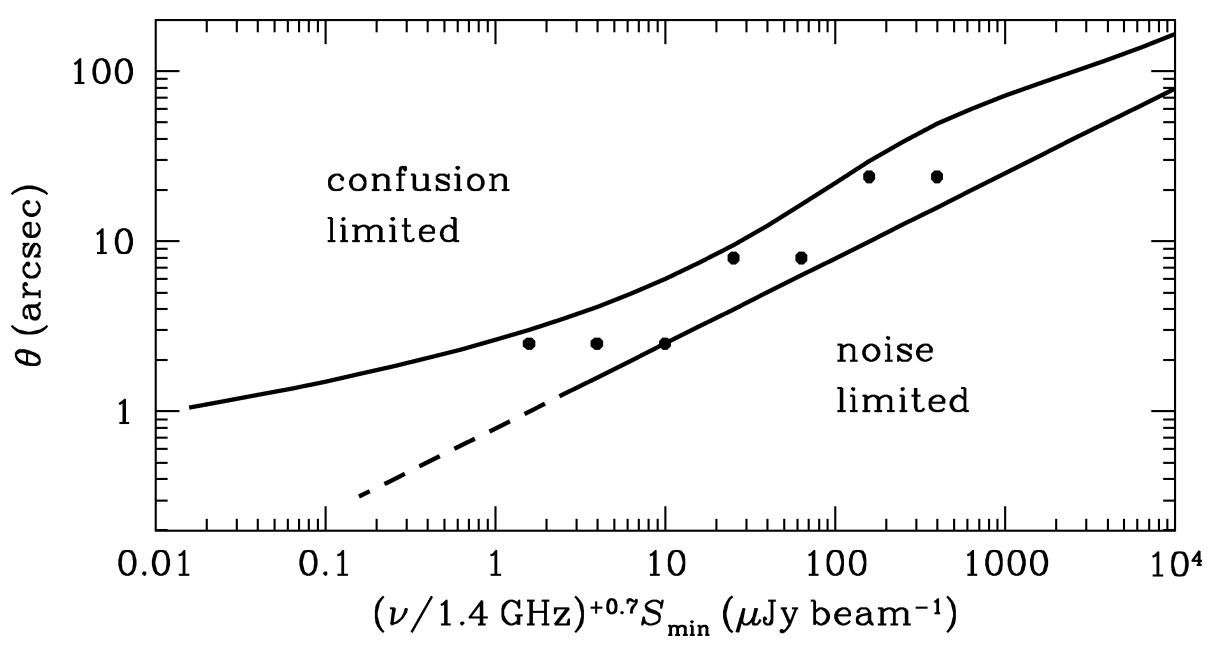

Figure 6: The $2.7 \mathrm{GHz}$ wedding cake survey described by Table 2 uses three VLA configurations with beamwidths $\theta(\operatorname{arcsec})=2.5,8$, and 24 to stay between the confusion and noise limits. The points show the $\left(S_{\min }, \theta\right)$ pairs of the seven layers.

rectangular grid divides the $L, z$ plane into 100 bins of height 0.4 in $\log (L)$ by width 0.135 in $\log (z)$. Low-luminosity high-redshift galaxies in the $\sim 25$ squares below the lowest dotted line will not be detected. The remaining 75 squares should contain the desired $N \sim 10^{2}$ galaxies each.

The integer number $N_{\mathrm{p}}$ of pointings needed for each layer to detect $\Delta N \sim 10^{3}$ galaxies with $S_{\min }<S<S_{\max }$ is shown in Column 5 , followed by the layer (on-source) integration time $\Delta \tau$ determined by the VLA exposure calculator assuming 25 working antennas, 8-bit sampling, a usable bandwidth of $1.5 \mathrm{GHz}$, and "robust" weighting. Notice that the configurations and sky coverages of Layer 1 and Layer 2 are the same, so Layers 1 and 2 can be merged. Likewise, Layer 1 or Layer 2 could replace one pointing in Layer 3, reducing the incremental time for Layer 3 from 66 to 44 hours, Layer 4 could replace 12 of the Layer 5 pointings, and Layer 6 could replace 91 of the Layer 7 pointings. The observing-time cost of the largest layers is so small that these layers could easily be increased in solid angle, at least up to the maximum solid angle covered by the necessary supporting data (deep optical or infrared identifications, and redshifts).

In this particular case, the most sensitive layer need cover only a single pointing, so it is no longer possible to trade off solid angle coverage for sensitivity. Consequently the deepest layer integration time is proportional to $S_{\min }^{-2}$, not $S_{\min }^{-1}$, and the deepest layer takes an even larger fraction of the total survey time. If $\sigma_{\mathrm{n}}=0.2 \mu \mathrm{Jy}_{\text {beam }}{ }^{-1}$, the total wedding-cake survey time is $\tau \approx 950$ hours. Increasing the noise limit to 0.30 or $0.40 \mu \mathrm{Jy} \mathrm{beam}^{-1}$ quickly lowers the total survey time to $\tau \approx 470$ or 300 hours, respectively. When $N_{\mathrm{p}}=1$ (Layer 1 of this survey), the Gaussian primary-beam attenuation of the VLA means that the top layer of the wedding cake has a Gaussian shape, not a flat one. Figure 8 shows the wedding cake for the 300-hour survey with $\sigma=0.4 \mu \mathrm{Jy}_{\text {beam }^{-1}}$.

At $S$ band, the VLA is sensitive enough to make the desired survey of SFRD evolution and can probably yield the required dynamic range if the deepest layer is placed in a field free of strong sources. L band ( $v \approx 1.4 \mathrm{GHz}$ ) is much more likely to be dynamic-range limited and is therefore not suitable. $\mathrm{C}$ band $(v \approx 6 \mathrm{GHz}, \Delta v \approx 3.4 \mathrm{GHz})$ would take $\sim 1.7 \times$ longer to detect the same 


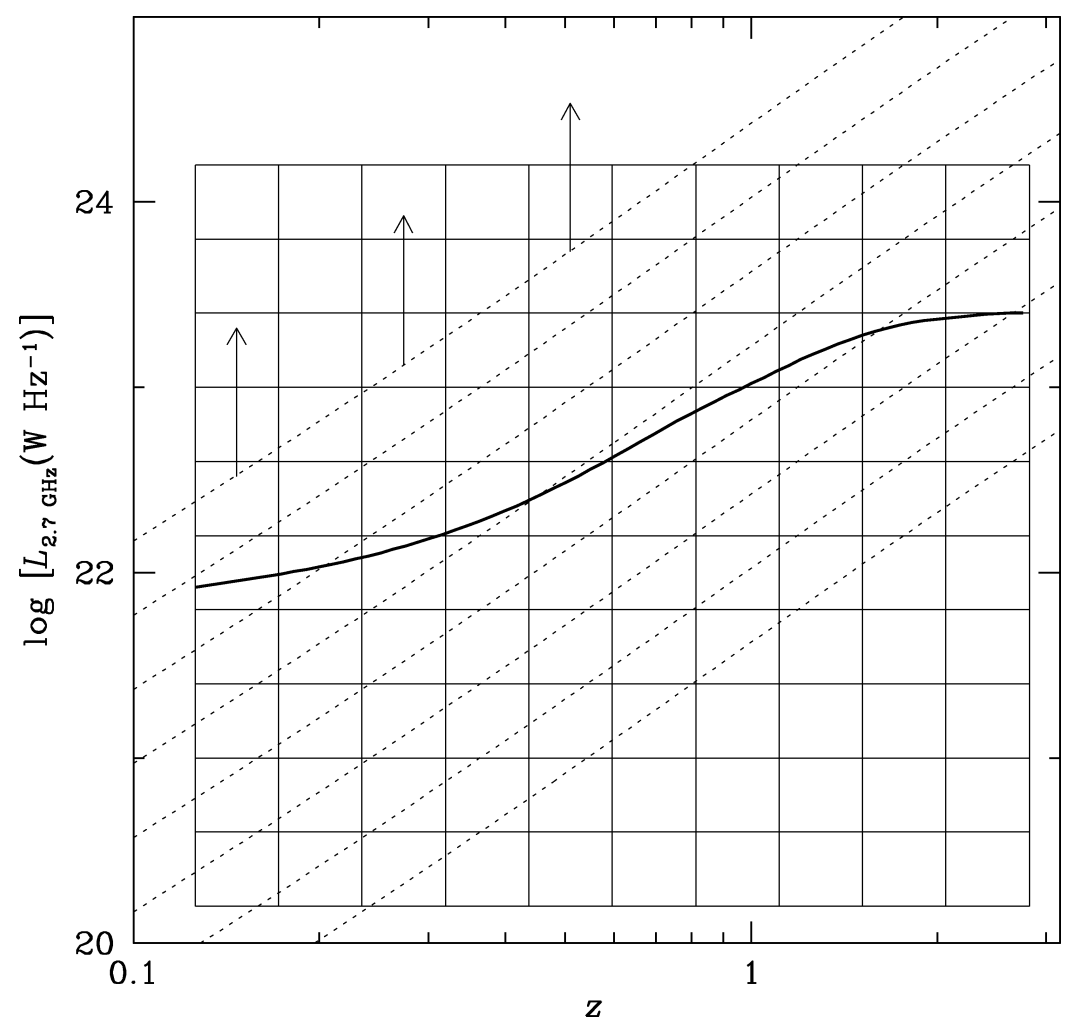

Figure 7: The luminosity-redshift $(L, z)$ plane of star-forming galaxies is mostly covered by the $2.7 \mathrm{GHz}$ wedding-cake survey whose layer flux-density limits (diagonal dotted lines) are specified in Table 2. The arrows above the highest diagonal line indicate that significant numbers of stronger sources will also be detected. The heavy curve is the evolving spectral luminosity at which $u_{\mathrm{m}}(L)$ and the SFRD are greatest. If the $(L, z)$ plane is divided into rectangles as shown, there should be $N \sim 10^{2}$ galaxies per square above the lowest diagonal line.

$\alpha=-0.7$ sources, and $\Delta N$ would be $\sim 5 \times$ smaller because the primary beam is smaller. Should the S-band option prove to be limited by dynamic range, the C-band alternative is marginally sensitive enough and is much less likely to be dynamic-range limited.

\section{References}

[1] Becker, R. H., R. L. White, \& D. J. Helfand, The FIRST survey: faint images of the radio sky at twenty centimeters, 1995, ApJ, 450:559

[2] Berta, S., B. Magnelli, R. Nordon et al., Building the cosmic infrared background brick by brick with Herschel/PEP, 2011, A\&A, 532A:49

[3] Condon, J. J., Confusion and flux-density error distributions, 1974, ApJ, 188:279

[4] Condon, J. J., Cosmological evolution of radio sources, 1984, ApJ, 287:461

[5] Condon, J. J., The 1.4 GHz luminosity function and its evolution, 1989, ApJ, 338:13

[6] Condon, J. J., Sensitive continuum surveys with the SKA: goals and challenges, 2009, SKA Memo 114, https://www.skatelescope.org/uploaded/12336_114_Memo_Condon.pdf 


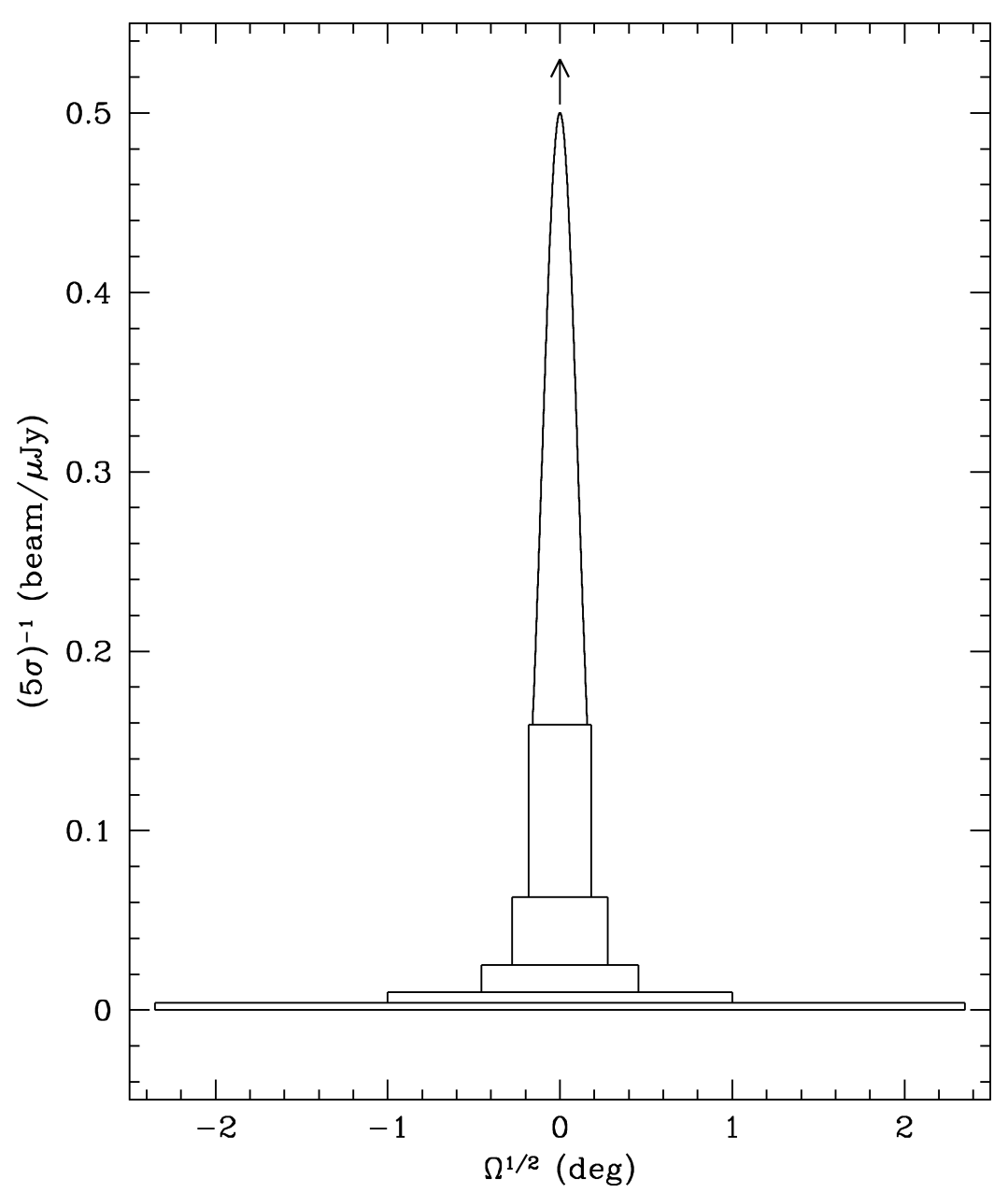

Figure 8: A seven-layer wedding cake designed to sample distant star-forming galaxy populations.

[7] Condon, J. J., W. D. Cotton, E. W. Greisen, Q. F. Yin, R. A. Perley, G. B. Taylor, \& J. J. Broderick, The NRAO VLA Sky Survey, 1998, AJ, 115:1693

[8] Condon, J. J., W. D. Cotton, \& J. J. Broderick, Radio sources and star formation in the local universe, 2002, AJ, 124:675

[9] Condon, J. J., W. D. Cotton, E. B. Fomalont, K. I. Kellermann, N. Miller, R. A. Perley, D. Scott, T. Vernstrom, \& J. V. Wall, Resolving the radio source background: deeper understanding through confusion, 2012, ApJ, 758:23

[10] Heywood, I., M. J. Jarvis, \& J. J. Condon, Sample variance, source clustering, and their influence on the counts of faint radio sources, 2013, MNRAS, 432:2625

[11] Hummel, E. The radio continuum properties of spiral galaxies, 1981, A\&A, 93:93

[12] Norris, R. P., A. M. Hopkins, J. Afonso et al., EMU: Evolutionary Map of the Universe, 2011, PASA, 28:215

[13] Wilman, R. J., L. Miller, M. J. Jarvis, T. Mauch, F. Levrier, F. B. Abdalla, S. Rqwlings, H.-R. Klöckner, D. Obreschkow, D. Olteanu, \& S. Young, A semi-empirical simulation of the extragalactic radio continuum sky for next generation radio telescopes, 2008, MNRAS, 388:1335 